ESAIM: PROCEEDINGS, May 2009, Vol. 27, p. 122-137

C. Besse, O. Goubet, T. Goudon \& S. Nicaise, Editors

\title{
TRAVEL TIME ESTIMATION BY CROSS CORRELATION OF NOISY SIGNALS
}

\author{
Josselin Garnier $^{1}$ and George Papanicolaou ${ }^{2}$
}

\begin{abstract}
In this review paper we consider the problem of estimating the singular support of the Green's function of the wave equation by using passive sensors. We assume that noise sources emit stationary random signals into the medium which are recorded by sensors. We show that the cross correlation of the recorded signals has enough information to identify the singular component of the Green's function, which provides an estimate of the travel time between the sensors. We consider different situations, such as when the support of the noise distribution extends over all space or is spatially limited, the medium is open or bounded, homogeneous or inhomogeneous, dissipative or not. We discuss the limitations of the cross correlation technique and identify configurations under which travel time estimation is possible. We show that iterated cross correlations using auxiliary sensors can be efficient for travel time estimation when the support of the noise source distribution is spatially limited.
\end{abstract}

\section{INTRODUCTION}

In this paper we consider the estimation of the Green's function of the wave equation in an inhomogeneous medium by cross correlation of noisy signals. We assume that noise sources with unknown spatial support emit stationary random signals, that propagate into the medium and are recorded at observation points. The cross correlation of the recorded signals has been shown to provide a reliable estimate of the Green's function and the travel time between the observation points in geophysics [22]. The travel time estimates can then be used for background velocity estimation. Indeed tomographic travel time velocity analysis, based on cross correlations, was applied successfully for surface-wave velocity estimation in Southern California [29], in Tibet [39], and in the Alps [36].

The idea of using the cross correlation of noisy signals to retrieve information about travel times was used previously in helioseismology and seismology $[18,25,32]$. It is now applied to seismic data from regional to local scales [21,22,34], volcano monitoring $[9,10,30]$ and petroleum prospecting [17]. When the noise sources are uncorrelated and have support that extends over all space, the derivative of the cross correlation of the recorded signals can be shown to be proportional to the symmetrized Green's function between the observation points [27]. This property also holds when the source distribution has limited spatial support provided the waves propagate in an ergodic cavity $[3,14,15]$. At the physical level this result has been established in other configurations provided that the noisy field is equipartitioned $[23,24,35,38]$. In an open environment this means that the recorded signals are an uncorrelated and isotropic superposition of plane waves in all directions. In a closed environment it means that the recorded signals are superpositions of normal modes with random amplitudes that are statistically uncorrelated and identically distributed. In this paper we introduce a mathematical formulation

\footnotetext{
${ }^{1}$ Laboratoire de Probabilités et Modèles Aléatoires \& Laboratoire Jacques-Louis Lions, Université Paris VII, 2 Place Jussieu, 75251 Paris Cedex 5

2 Mathematics Department, Stanford University, Stanford, CA 94305
}

(C) EDP Sciences, SMAI 2009 
in which travel time estimation by cross correlation of noisy signals is possible when there is enough noise source diversity.

In many realistic environments the noise source distribution is spatially limited and the field is not equipartitioned. As a result, the waves recorded by the observation points are dominated by the flux coming from the direction of the noise sources, which results in an azimuthal dependence of the quality of the Green's function estimation, with poor results for some azimuths [36]. To overcome this problem, Campillo and Stehly [12] have recently proposed the use higher-order cross correlations. In this paper, we first explain why the usual cross correlation technique fails when the noise sources have limited spatial support. We then show that iterated cross correlations using auxiliary observation points can exploit the enhanced directional diversity of the waves scattered by the heterogeneities of the medium. We analyze a special fourth-order cross correlation function that can provide acceptable travel time estimates even when the support of the noise sources is spatially limited.

The paper is organized as follows. In Section 2 we describe the physical principles for travel time estimation by cross correlation of noisy signals. In Section 3 we present a mathematical formulation of the estimation problem. In Sections 4 we give a simple proof of the relation between the cross correlation and the Green's function when the sources are distributed all over space. In Section 5 we present the Helmholtz-Kirchhoff theorem and its application to cross correlations when the noise sources completely surround the region under investigation. In Sections 4-5 it is sufficient to assume that the recording time is much larger than the coherence time of the sources and then the full Green's function can be estimated. When the noise sources have a spatially limited distribution, the singular (high-frequency) component of the Green's function can still be estimated provided that some additional conditions are fulfilled. As a result travel time estimation is still possible provided that the typical travel time is much larger than the coherence time of the noise sources. In Section 6 we give conditions under which travel time estimation by cross correlations is possible in an open medium when the noise sources are spatially localized, using stationary phase analysis. In Section 7 we show that travel time estimation in an ergodic cavity is possible even when the sources are spatially localized, using semi-classical analysis. In Section 8 we study properties of iterated cross correlations, which requires the analysis of fluctuations of cross correlations due to heterogeneities in the medium, and we show that travel time estimation can be done with iterated cross correlations even when the spatial support of the noise source distribution is limited.

\section{TRAVEL TIME ESTIMATION With CROSS CORRELATIONS}

In this section we present the physical context that motivates travel time estimation with cross correlations, and discuss the limitations of this approach.

We would like to reconstruct the background velocity of the earth's crust. The usual technique for this is to wait for an earthquake to occur, which plays the role of a source, and to record the signals (seismograms) at various observation points. Travel time estimation is done using the recorded direct arrivals and then, if enough observation points are available, it is possible to construct a map of the background velocity. The direct arrivals correspond to ballistic waves that propagate along rays from the sources to the observation points. After the direct arrivals, the seismograms are long oscillatory signals with decreasing amplitude but still above the noise level. These signals correspond to coda waves that are scattered by the heterogeneities of the earth crust. Coda waves have been analyzed because they contain information about the medium [2,31]. It was understood only very recently that the background noise (the stationary, noisy signals recorded during the long time intervals between earthquakes) also contains information about the medium. The issue is then how to extract this information, which is not as easy as travel time estimation from direct arrivals.

The noise signals recorded over time intervals between earthquakes have components due to surface waves generated from the interaction of the ocean swell with the coast [36]. The medium in which the waves propagate has a slowly varying background velocity profile, which determines the travel times that we want to estimate, as well as heterogeneities that are responsible for wave scattering. It was proposed in $[18,25]$ to compute the cross correlation (in time) of the noisy signals $u\left(t, \mathbf{x}_{1}\right)$ and $u\left(t, \mathbf{x}_{2}\right)$ recorded at two observation points $\mathbf{x}_{1}$ and 

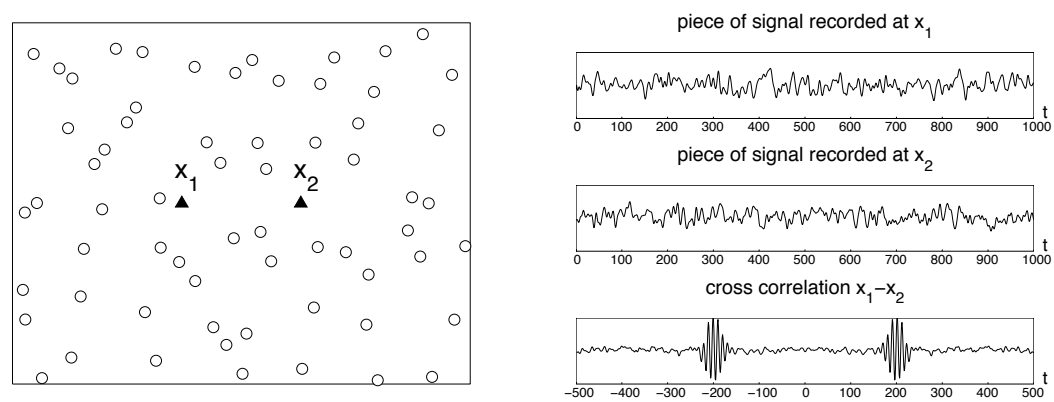

FiguRE 1. When the spatial support of the noise sources (circles) extends over all space then the cross correlation function is symmetric. Its positive and negative parts correspond to the Green's function between $\mathbf{x}_{1}$ and $\mathbf{x}_{2}$ and its anti-causal form, respectively.

$\mathbf{x}_{2}$ :

$$
C_{T}\left(\tau, \mathbf{x}_{1}, \mathbf{x}_{2}\right)=\frac{1}{T} \int_{0}^{T} u\left(t, \mathbf{x}_{1}\right) u\left(t+\tau, \mathbf{x}_{2}\right) d t .
$$

In a homogeneous medium, if the source of the waves is a space-time stationary random field that is also delta correlated in space and in time then it can been shown $[27,35]$ that

$$
\frac{\partial}{\partial \tau} C_{T}\left(\tau, \mathbf{x}_{1}, \mathbf{x}_{2}\right) \simeq G\left(\tau, \mathbf{x}_{1}, \mathbf{x}_{2}\right)-G\left(-\tau, \mathbf{x}_{1}, \mathbf{x}_{2}\right),
$$

where $G$ is the time-domain Green's function of the wave propagation process. This approximate equality holds for $T$ sufficiently large, provided some limiting absorption is introduced to regularize the integral. When the medium is homogeneous, a mathematical analysis of (2) is given in Section 4. When the medium is inhomogeneous and the sources surround the inhomogeneous region of interest, then (2) still holds, as can be shown by the Kirchhoff-Helmholtz theorem [37] that we present in Section 5. The main point here is that the time-symmetrized Green's function can be obtained from the cross correlation if there is enough source diversity. In this case the wave field at any sensor is essentially equipartitioned, in the sense that it is a superposition of uncorrelated plane waves in all directions. The travel time between $\mathbf{x}_{1}$ and $\mathbf{x}_{2}$ can be obtained from the singular support of the cross correlation.

The configuration (Figure 1) in which the spatial support of the noise sources extends over all space is rarely encountered in applications. Significant departures from this ideal situation occur when limited spatial diversity of the sources introduces directivity into the recorded fields, which affects the quality of the estimate of the Green's function. If, in particular, the source distribution is spatially localized, then the flux of wave energy is not isotropic, and the cross correlation function is not symmetric (Figure 2). In some situations it may be impossible to distinguish the coherent part of the cross correlation function, which contains information about the travel time (Figure 3). A mathematical analysis using the stationary phase method is given in Section 6 .

In the case of a spatially localized distribution of noise sources, directional diversity of the recorded fields can be enhanced if there is sufficient scattering in the medium. An ergodic cavity with a homogeneous or inhomogeneous interior is a good example (Figure 4, left): Even with a source distribution that has very limited spatial support, the reverberations of the waves in the cavity generate interior fields with high directional diversity $[3,14]$. We analyze this situation in Section 7 . Multiple scattering of waves by random inhomogeneities can also lead to wave field equipartition if the transport mean free path is short compared to the distance from the sources to the sensors $[22,28,31]$. The transport mean free path is the propagation distance over which wave energy transport in a scattering medium is effectively isotropic. In such a scattering medium (Figure 4, right), 

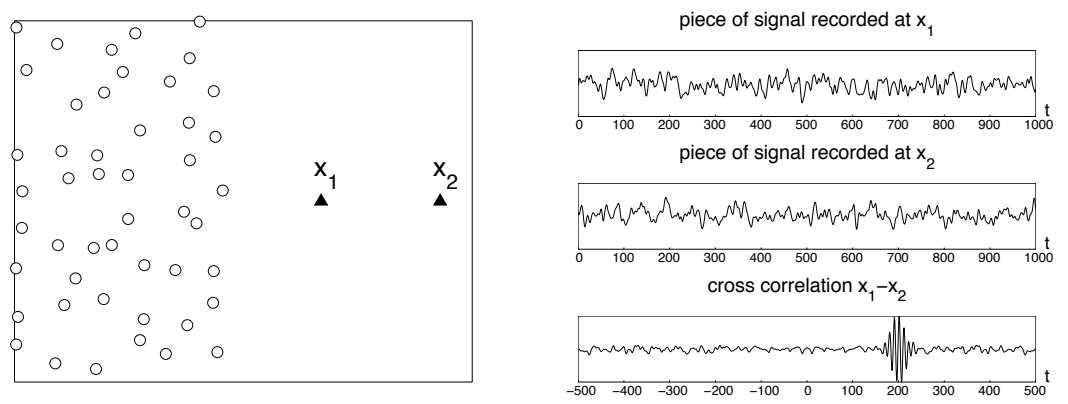

FiguRE 2. When the distribution of noise sources is spatially localized then the cross correlation function is not symmetric.

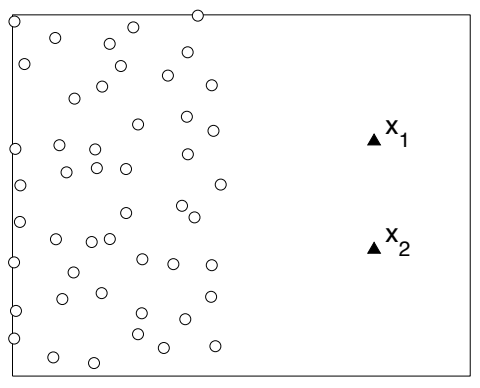

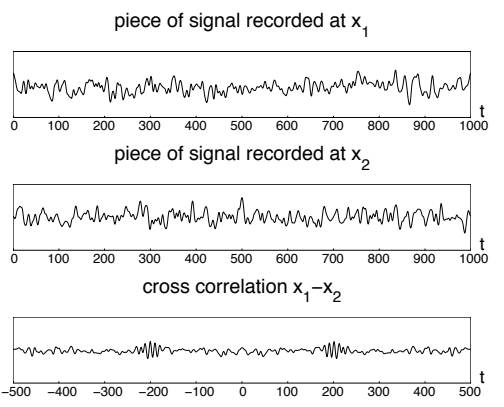

FIGURE 3. When the distribution of noise sources is spatially localized then the coherent part of the cross correlation function can be difficult or even impossible to distinguish if the axis formed by the two sensors is perpendicular to the main direction of energy flux from the noise sources.

the inhomogeneities can be viewed as secondary sources in the vicinity of the sensors. In Section 8 we describe how to exploit the enhanced directional diversity of the scattered waves.

The role of scattering in a random medium for travel time estimation depends on the transport mean free path. We have just seen that directional diversity is enhanced provided that the transport mean free path is short compared to the distance between the sources and the sensors. If the transport mean free path is short compared to the distance between the sensors, then the cross correlation function gives an acceptable estimate of the Green's function, which is random because of the medium. The coherent part of the Green's function that has information about the travel time is essentially unobservable. Therefore, when the noise sources are spatially limited then the travel time can be estimated in a random medium provided that (i) the transport mean free path is short compared to the distance between the sources and the sensors, and (ii) it is long compared to the distance between the sensors. This is the physical situation in which the random inhomogeneities actually enhance the estimation process. 

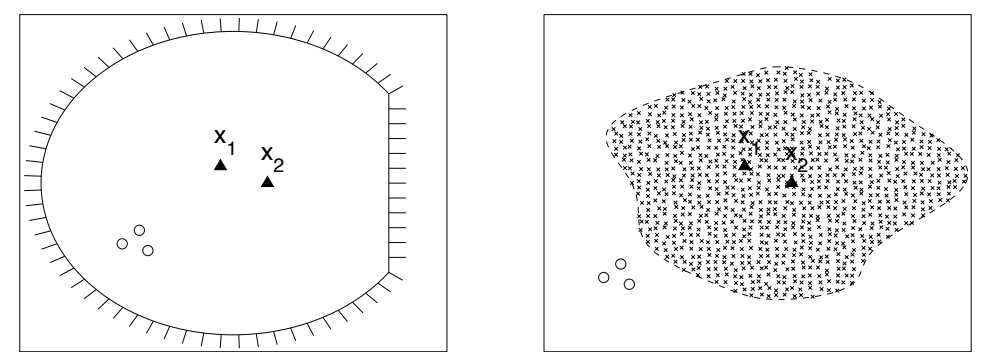

Figure 4. Configurations in which wave fields have directional diversity. An ergodic cavity (left figure) and a randomly inhomogeneous medium (right figure).

\section{ThE EMPIRICAL CROSS CORRELATION AND THE STATISTICAL CROSS CORRELATION}

We consider the solution $u$ of the wave equation in a $d$-dimensional inhomogeneous medium:

$$
\frac{1}{c^{2}(\mathbf{x})} \frac{\partial^{2} u}{\partial t^{2}}-\Delta_{\mathbf{x}} u=n^{\varepsilon}(t, \mathbf{x})
$$

The domain can be bounded, with prescribed boundary conditions at the boundary, or unbounded, in which case the support of the inhomogeneous region is assumed to be compactly supported. The term $n^{\varepsilon}(t, \mathbf{x})$ models a random distribution of noise sources. It is a zero-mean stationary (in time) Gaussian process with autocorrelation function

$$
\left\langle n^{\varepsilon}\left(t_{1}, \mathbf{y}_{1}\right) n^{\varepsilon}\left(t_{2}, \mathbf{y}_{2}\right)\right\rangle=F^{\varepsilon}\left(t_{2}-t_{1}\right) \Gamma^{\varepsilon}\left(\mathbf{y}_{1}, \mathbf{y}_{2}\right) .
$$

Here $\langle\cdot\rangle$ stands for statistical average with respect to the distribution of the noise sources. The parameter $\varepsilon$ denotes the ratio of the decoherence time of the noise sources over the typical travel time between sensors. In the first sections of this paper, $\varepsilon$ can be arbitrary. In Sections 6-8 we assume that $\varepsilon$ is small and carry out an asymptotic analysis using this hypothesis. We can then write the time correlation function $F^{\varepsilon}$ in the form

$$
F^{\varepsilon}\left(t_{2}-t_{1}\right)=F\left(\frac{t_{2}-t_{1}}{\varepsilon}\right)
$$

where $t_{1}$ and $t_{2}$ are scaled relative to typical sensor travel times. The Fourier transform $\hat{F}^{\varepsilon}$ of the time correlation function is a nonnegative, even real-valued function. It is proportional to the power spectral density of the sources:

with the Fourier transform defined by

$$
\hat{F}^{\varepsilon}(\omega)=\varepsilon \hat{F}(\varepsilon \omega),
$$

$$
\hat{F}(\omega)=\int F(t) e^{i \omega t} d t .
$$

The spatial distribution of the noise sources is characterized by the auto-covariance function $\Gamma^{\varepsilon}$. It is the kernel of a symmetric nonnegative definite operator. For simplicity, we assume in the first sections of this paper that the process $n^{\varepsilon}$ is delta-correlated in space:

$$
\Gamma^{\varepsilon}\left(\mathbf{y}_{1}, \mathbf{y}_{2}\right)=\theta\left(\mathbf{y}_{1}\right) \delta\left(\mathbf{y}_{1}-\mathbf{y}_{2}\right),
$$

where $\theta$ characterizes the spatial support of the sources. One can consider a more general form for the spatial auto-covariance function as is done in Section 7. This requires the use of semiclassical analysis, but the results do not change qualitatively. 
The stationary solution of the wave equation has the integral representation

$$
u(t, \mathbf{x})=\iint G(s, \mathbf{x}, \mathbf{y}) n^{\varepsilon}(t-s, \mathbf{y}) d s d \mathbf{y},
$$

where $G(t, \mathbf{x}, \mathbf{y})$ is the time-dependent Green's function. It is the fundamental solution of the wave equation

$$
\frac{1}{c^{2}(\mathbf{x})} \frac{\partial^{2} G}{\partial t^{2}}-\Delta_{\mathbf{x}} G=\delta(t) \delta(\mathbf{x}-\mathbf{y})
$$

starting from $G(0, \mathbf{x}, \mathbf{y})=\partial_{t} G(0, \mathbf{x}, \mathbf{y})=0$ (and extended to the negative time axis by $\left.G(t, \mathbf{x}, \mathbf{y})=0 \forall t \leq 0\right)$.

The empirical cross correlation of the signals recorded at $\mathbf{x}_{1}$ and $\mathbf{x}_{2}$ for an integration time $T$ is defined by (1). It is a statistically stable quantity, in the sense that for a large integration time $T, C_{T}$ is independent of the realization of the noise sources. This is stated in the following proposition proved in [20].

Proposition 3.1. 1. The expectation of $C_{T}$ (with respect to the noise source distribution) is independent of $T$ :

$$
\left\langle C_{T}\left(\tau, \mathbf{x}_{1}, \mathbf{x}_{2}\right)\right\rangle=C^{(1)}\left(\tau, \mathbf{x}_{1}, \mathbf{x}_{2}\right),
$$

where $C^{(1)}$ is given by

$$
C^{(1)}\left(\tau, \mathbf{x}_{1}, \mathbf{x}_{2}\right)=\int d \mathbf{y} \iint d s d s^{\prime} G\left(s, \mathbf{x}_{1}, \mathbf{y}\right) G\left(\tau+s+s^{\prime}, \mathbf{x}_{2}, \mathbf{y}\right) F^{\varepsilon}\left(s^{\prime}\right) \theta(\mathbf{y}),
$$

or equivalently by

$$
C^{(1)}\left(\tau, \mathbf{x}_{1}, \mathbf{x}_{2}\right)=\int d \mathbf{y} \int d \omega \overline{\hat{G}}\left(\omega, \mathbf{x}_{1}, \mathbf{y}\right) \hat{G}\left(\omega, \mathbf{x}_{2}, \mathbf{y}\right) \hat{F}^{\varepsilon}(\omega) e^{-i \omega \tau} \theta(\mathbf{y})
$$

2. The empirical cross correlation $C_{T}$ is a self-averaging quantity:

$$
C_{T}\left(\tau, \mathbf{x}_{1}, \mathbf{x}_{2}\right) \stackrel{T \rightarrow \infty}{\longrightarrow} C^{(1)}\left(\tau, \mathbf{x}_{1}, \mathbf{x}_{2}\right)
$$

in probability with respect to the distribution of the sources. More precisely, the fluctuations of $C_{T}$ around its mean value $C^{(1)}$ are of order $T^{-1 / 2}$.

In this paper, we shall always assume that the integration time $T$ is large enough so that the empirical cross correlation $C_{T}$ can be considered as equal to the statistical cross correlation $C^{(1)}$.

\section{Emergence of the Green's Function FOR an EXTEnded Distribution of SOURCES IN A HOMOGENEOUS MEDIUM}

We give an elementary proof of the relation between the cross correlation and the Green's function when the medium is homogeneous and open with background velocity $c_{0}$, and the source distribution extends over all space, i.e. $\theta \equiv 1$, as in Figure 1. In this case the signal amplitude diverges because the contributions from the noise sources far away from the sensors are not damped. For a well-posed formulation we need to introduce some dissipation, so we consider the solution $u$ of the damped wave equation:

$$
\frac{1}{c_{0}^{2}}\left(\frac{1}{T_{a}}+\frac{\partial}{\partial t}\right)^{2} u-\Delta_{\mathbf{x}} u=n^{\varepsilon}(t, \mathbf{x})
$$

The following proposition can be found in [20]. A somewhat different form, with delta-correlated in time sources and with a different definition of dissipation, can be found in [27]. 
Proposition 4.1. In a three-dimensional open medium with dissipation, if the source distribution extends over all space $\theta \equiv 1$, then

$$
\frac{\partial}{\partial \tau} C^{(1)}\left(\tau, \mathbf{x}_{1}, \mathbf{x}_{2}\right)=-\frac{c_{0}^{2} T_{a}}{4} e^{-\frac{\left|\mathbf{x}_{1}-\mathbf{x}_{2}\right|}{c_{0} T_{a}}}\left[F^{\varepsilon} * G\left(\tau, \mathbf{x}_{1}, \mathbf{x}_{2}\right)-F^{\varepsilon} * G\left(-\tau, \mathbf{x}_{1}, \mathbf{x}_{2}\right)\right]
$$

where * stands for the convolution in $\tau$ and $G$ is the Green's function of the homogeneous medium without dissipation:

$$
G\left(t, \mathbf{x}_{1}, \mathbf{x}_{2}\right)=\frac{1}{4 \pi\left|\mathbf{x}_{1}-\mathbf{x}_{2}\right|} \delta\left(t-\frac{\left|\mathbf{x}_{1}-\mathbf{x}_{2}\right|}{c_{0}}\right) .
$$

If the decoherence time of the sources is much shorter than the travel time (i.e., $\varepsilon \ll 1$ ), then $F^{\varepsilon}$ behaves like a Dirac distribution in (16) and we have

$$
\frac{\partial}{\partial \tau} C^{(1)}\left(\tau, \mathbf{x}_{1}, \mathbf{x}_{2}\right) \simeq e^{-\frac{\left|\mathbf{x}_{1}-\mathbf{x}_{2}\right|}{c_{0} T_{a}}}\left[G\left(\tau, \mathbf{x}_{1}, \mathbf{x}_{2}\right)-G\left(-\tau, \mathbf{x}_{1}, \mathbf{x}_{2}\right)\right]
$$

up to a multiplicative constant. It is therefore possible to estimate the travel time $\tau\left(\mathbf{x}_{1}, \mathbf{x}_{2}\right)=\left|\mathbf{x}_{1}-\mathbf{x}_{2}\right| / c_{0}$ between $\mathbf{x}_{1}$ and $\mathbf{x}_{2}$ from the cross correlation, with an accuracy of the order of the decoherence time of the noise sources.

Proof. The Green's function of the homogeneous medium with dissipation is: $G_{a}\left(t, \mathbf{x}_{1}, \mathbf{x}_{2}\right)=G\left(t, \mathbf{x}_{1}, \mathbf{x}_{2}\right) e^{-\frac{t}{T_{a}}}$. The cross correlation function is given by (12):

$$
C^{(1)}\left(\tau, \mathbf{x}_{1}, \mathbf{x}_{2}\right)=\int d \mathbf{y} \iint d s d s^{\prime} G_{a}\left(s, \mathbf{x}_{1}, \mathbf{y}\right) G_{a}\left(\tau+s+s^{\prime}, \mathbf{x}_{2}, \mathbf{y}\right) F^{\varepsilon}\left(s^{\prime}\right) .
$$

Integrating in $s$ and $s^{\prime}$ gives

$$
C^{(1)}\left(\tau, \mathbf{x}_{1}, \mathbf{x}_{2}\right)=\int \frac{d \mathbf{y}}{16 \pi^{2}\left|\mathbf{x}_{1}-\mathbf{y}\right|\left|\mathbf{x}_{2}-\mathbf{y}\right|} e^{-\frac{\left|\mathbf{x}_{1}-\mathbf{y}\right|+\left|\mathbf{x}_{2}-\mathbf{y}\right|}{c_{0} T_{a}}} F^{\varepsilon}\left(\tau-\frac{\left|\mathbf{x}_{1}-\mathbf{y}\right|-\left|\mathbf{x}_{2}-\mathbf{y}\right|}{c_{0}}\right) .
$$

We parameterize the locations of the sensors by $\mathbf{x}_{1}=(h, 0,0)$ and $\mathbf{x}_{2}=(-h, 0,0)$, where $h>0$, and we use the change of variables for $\mathbf{y}=(x, y, z)$ :

$$
\begin{cases}x=h \sin \theta \cosh \phi, & \phi \in(0, \infty) \\ y=h \cos \theta \sinh \phi \cos \psi, & \theta \in(-\pi / 2, \pi / 2) \\ z=h \cos \theta \sinh \phi \sin \psi, & \psi \in(0,2 \pi)\end{cases}
$$

whose Jacobian is $J=h^{3} \cos \theta \sinh \phi\left(\cosh ^{2} \phi-\sin ^{2} \theta\right)$. Using the fact that $\left|\mathbf{x}_{1}-\mathbf{y}\right|=h(\cosh \phi-\sin \theta)$ and $\left|\mathbf{x}_{2}-\mathbf{y}\right|=h(\cosh \phi+\sin \theta)$, we get

$$
C^{(1)}\left(\tau, \mathbf{x}_{1}, \mathbf{x}_{2}\right)=\frac{h}{8 \pi} \int_{0}^{\infty} d \phi \sinh \phi \int_{-\pi / 2}^{\pi / 2} d \theta \cos \theta e^{-\frac{2 h \cosh \phi}{c_{0} T_{a}}} F^{\varepsilon}\left(\tau+\frac{2 h \sin \theta}{c_{0}}\right) .
$$

After the new change of variables $u=h \cosh \phi$ and $s=\left(2 h / c_{0}\right) \sin \theta$, we obtain

$$
C^{(1)}\left(\tau, \mathbf{x}_{1}, \mathbf{x}_{2}\right)=\frac{c_{0}^{2} T_{a}}{32 \pi h} e^{-\frac{2 h}{c_{0} T_{a}}} \int_{-2 h / c_{0}}^{2 h / c_{0}} d s F^{\varepsilon}(\tau+s)
$$

By differentiating in $\tau$, we get

$$
\frac{\partial}{\partial \tau} C^{(1)}\left(\tau, \mathbf{x}_{1}, \mathbf{x}_{2}\right)=\frac{c_{0}^{2} T_{a}}{32 \pi h} e^{-\frac{2 h}{c_{0} T_{a}}}\left[F^{\varepsilon}\left(\tau+\frac{2 h}{c_{0}}\right)-F^{\varepsilon}\left(\tau-\frac{2 h}{c_{0}}\right)\right],
$$




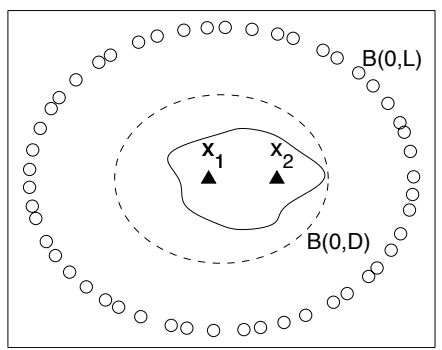

Figure 5. The sources are distributed on the sphere $\partial B(\mathbf{0}, L)$ and the medium is homogeneous outside the ball $B(\mathbf{0}, D)$.

which is the desired result since $\left|\mathbf{x}_{1}-\mathbf{x}_{2}\right|=2 h$.

\section{Emergence of the Green's FUnCTION FOR AN EXTENDED Distribution of SOURCES IN AN INHOMOGENEOUS MEDIUM}

The cross correlation function is closely related to the symmetrized Green's function from $\mathbf{x}_{1}$ to $\mathbf{x}_{2}$ not only for a homogeneous medium but also for an inhomogeneous medium, as discussed in the introduction. Here we give a simple and rigorous proof for an open inhomogeneous medium in the case in which the noise sources are located on the surface of a sphere that encloses both the inhomogeneous region and the sensors, located at $\mathbf{x}_{1}$ and $\mathbf{x}_{2}$ (Figure 5). The proof is based on an approximate identity that follows from Green's identity and the Sommerfeld radiation condition. This approximate identity can be viewed as a version of the HelmholtzKirchhoff integral theorem (known in acoustics [6, p. 473] and in optics [8, p. 419]) and it is also presented in [37].

Proposition 5.1. Let us assume that the medium is homogeneous with background velocity $c_{e}$ outside the ball $B(\mathbf{0}, D)$ with center $\mathbf{0}$ and radius $D$. Then, for any $\mathbf{x}_{1}, \mathbf{x}_{2} \in B(\mathbf{0}, D)$ we have for $L \gg D$ :

$$
\hat{G}\left(\omega, \mathbf{x}_{1}, \mathbf{x}_{2}\right)-\overline{\hat{G}}\left(\omega, \mathbf{x}_{1}, \mathbf{x}_{2}\right)=\frac{2 i \omega}{c_{e}} \int_{\partial B(\mathbf{0}, L)} \overline{\hat{G}}\left(\omega, \mathbf{x}_{1}, \mathbf{y}\right) \hat{G}\left(\omega, \mathbf{x}_{2}, \mathbf{y}\right) d S(\mathbf{y}) .
$$

Proof. We consider the equation satisfied by the time-harmonic Green's function with the source at $\mathbf{x}_{2}$ and the complex conjugate form of this equation with the source at $\mathbf{x}_{1}$ :

$$
\Delta_{\mathbf{y}} \hat{G}\left(\omega, \mathbf{y}, \mathbf{x}_{2}\right)+\frac{\omega^{2}}{c^{2}(\mathbf{y})} \hat{G}\left(\omega, \mathbf{y}, \mathbf{x}_{2}\right)=-\delta\left(\mathbf{y}-\mathbf{x}_{2}\right), \quad \Delta_{\mathbf{y}} \overline{\hat{G}}\left(\omega, \mathbf{y}, \mathbf{x}_{1}\right)+\frac{\omega^{2}}{c^{2}(\mathbf{y})} \overline{\hat{G}}\left(\omega, \mathbf{y}, \mathbf{x}_{1}\right)=-\delta\left(\mathbf{y}-\mathbf{x}_{1}\right) .
$$

We multiply the first equation by $\bar{G}\left(\omega, \mathbf{y}, \mathbf{x}_{1}\right)$ and subtract the second equation multiplied by $\hat{G}\left(\omega, \mathbf{y}, \mathbf{x}_{2}\right)$ :

$$
\begin{array}{r}
\nabla_{\mathbf{y}} \cdot\left[\hat{\hat{G}}\left(\omega, \mathbf{y}, \mathbf{x}_{1}\right) \nabla_{\mathbf{y}} \hat{G}\left(\omega, \mathbf{y}, \mathbf{x}_{2}\right)-\hat{G}\left(\omega, \mathbf{y}, \mathbf{x}_{2}\right) \nabla_{\mathbf{y}} \hat{\hat{G}}\left(\omega, \mathbf{y}, \mathbf{x}_{1}\right)\right]=\hat{G}\left(\omega, \mathbf{y}, \mathbf{x}_{2}\right) \delta\left(\mathbf{y}-\mathbf{x}_{1}\right)-\overline{\hat{G}}\left(\omega, \mathbf{y}, \mathbf{x}_{1}\right) \delta\left(\mathbf{y}-\mathbf{x}_{2}\right) \\
=\hat{G}\left(\omega, \mathbf{x}_{1}, \mathbf{x}_{2}\right) \delta\left(\mathbf{y}-\mathbf{x}_{1}\right)-\overline{\hat{G}}\left(\omega, \mathbf{x}_{1}, \mathbf{x}_{2}\right) \delta\left(\mathbf{y}-\mathbf{x}_{2}\right),
\end{array}
$$

where we have used the reciprocity property $\hat{G}\left(\omega, \mathbf{x}_{2}, \mathbf{x}_{1}\right)=\hat{G}\left(\omega, \mathbf{x}_{1}, \mathbf{x}_{2}\right)$. We next integrate over the ball $B(\mathbf{0}, L)$ and use the divergence theorem:

$$
\int_{\partial B(\mathbf{0}, L)} \mathbf{n}(\mathbf{y}) \cdot\left[\overline{\hat{G}}\left(\omega, \mathbf{y}, \mathbf{x}_{1}\right) \nabla_{\mathbf{y}} \hat{G}\left(\omega, \mathbf{y}, \mathbf{x}_{2}\right)-\hat{G}\left(\omega, \mathbf{y}, \mathbf{x}_{2}\right) \nabla_{\mathbf{y}} \overline{\hat{G}}\left(\omega, \mathbf{y}, \mathbf{x}_{1}\right)\right] d S(\mathbf{y})=\hat{G}\left(\omega, \mathbf{x}_{1}, \mathbf{x}_{2}\right)-\overline{\hat{G}}\left(\omega, \mathbf{x}_{1}, \mathbf{x}_{2}\right),
$$


where $\mathbf{n}(\mathbf{y})$ is the unit outward normal to the ball $B(\mathbf{0}, L)$, which is $\mathbf{n}(\mathbf{y})=\mathbf{y} /|\mathbf{y}|$. This relation can be viewed as the second Green's identity. The Green's function also satisfies the Sommerfeld radiation condition

$$
\lim _{|\mathbf{y}| \rightarrow \infty}|\mathbf{y}|^{\frac{d-1}{2}}\left(\frac{\mathbf{y}}{|\mathbf{y}|} \cdot \nabla_{\mathbf{y}}-i \frac{\omega}{c_{e}}\right) \hat{G}\left(\omega, \mathbf{y}, \mathbf{x}_{1}\right)=0
$$

uniformly in all directions $\mathbf{y} /|\mathbf{y}|$. Under the conditions stated in the proposition, we can substitute $i\left(\omega / c_{e}\right) \hat{G}\left(\omega, \mathbf{y}, \mathbf{x}_{2}\right)$ for $\mathbf{n} \cdot \nabla_{\mathbf{y}} \hat{G}\left(\omega, \mathbf{y}, \mathbf{x}_{2}\right)$ in the surface integral over $\partial B(\mathbf{0}, L)$, and $-i\left(\omega / c_{e}\right) \overline{\hat{G}}\left(\omega, \mathbf{y}, \mathbf{x}_{1}\right)$ for $\mathbf{n} \cdot \nabla_{\mathbf{y}} \overline{\hat{G}}\left(\omega, \mathbf{y}, \mathbf{x}_{1}\right)$, which gives the desired result. Note that it is important that the medium be homogeneous in the exterior of the ball $B(\mathbf{0}, D)$ in order be able to use the radiation condition.

The right side of the Helmholtz-Kirchhoff identity (17) is related to the representation (13) of the cross correlation function $C^{(1)}$ in the Fourier domain. Therefore, by substituting (17) into (13) we get the following corollary.

Corollary 5.2. We assume that

1) the medium is homogeneous outside the ball $B(\mathbf{0}, D)$ with center $\mathbf{0}$ and radius $D$,

2) the sources are localized with a uniform density on the sphere $\partial B(\mathbf{0}, L)$ with center $\mathbf{0}$ and radius $L$.

If $L \gg D$, then for any $\mathbf{x}_{1}, \mathbf{x}_{2} \in B(\mathbf{0}, D)$, we have (up to a multiplicative factor)

$$
\frac{\partial}{\partial \tau} C^{(1)}\left(\tau, \mathbf{x}_{1}, \mathbf{x}_{2}\right)=F^{\varepsilon} * G\left(\tau, \mathbf{x}_{1}, \mathbf{x}_{2}\right)-F^{\varepsilon} * G\left(-\tau, \mathbf{x}_{1}, \mathbf{x}_{2}\right) .
$$

If in addition we have $\varepsilon \ll 1$, then $F^{\varepsilon}$ behaves approximately like a delta distribution acting on the Green's function and we get (2).

\section{TRAVEL Time ESTIMATION With SPATIALLY LOCALIZED NOISE SOURCES IN AN OPEN} MEDIUM

We study in this section the cross correlation function when the support of the sources is spatially limited in an open non-dissipative medium. We assume that the fluctuations of the medium parameters are modeled by a smooth background velocity profile $c_{0}(\mathbf{x})$, which is homogeneous outside a large sphere that encloses the sensors and the sources. The outgoing time-harmonic Green's function $\hat{G}_{0}$ of the medium is the solution of

$$
\Delta_{\mathbf{x}} \hat{G}_{0}(\omega, \mathbf{x}, \mathbf{y})+\frac{\omega^{2}}{c_{0}^{2}(\mathbf{x})} \hat{G}_{0}(\omega, \mathbf{x}, \mathbf{y})=-\delta(\mathbf{x}-\mathbf{y}),
$$

along with the radiation condition at infinity. When the background is homogeneous with constant wave speed $c_{0}$ then the homogeneous outgoing time-harmonic Green's function is

$$
\hat{G}_{0}(\omega, \mathbf{x}, \mathbf{y})=\frac{e^{i \omega \frac{|\mathbf{y}-\mathbf{x}|}{c_{0}}}}{4 \pi|\mathbf{y}-\mathbf{x}|}
$$

in three-dimensional space, and

$$
\hat{G}_{0}(\omega, \mathbf{x}, \mathbf{y})=\frac{i}{4} H_{0}^{(1)}\left(\omega \frac{|\mathbf{y}-\mathbf{x}|}{c_{0}}\right)
$$

in two-dimensional space. Here $H_{0}^{(1)}$ is the zeroth order Hankel function of the first kind. Using the asymptotic form of the Hankel function [1, formula 9.2.3], we see that the high-frequency behavior of the Green's function is related to the homogeneous medium travel time $|\mathbf{x}-\mathbf{y}| / c_{0}$ :

$$
\hat{G}_{0}\left(\frac{\omega}{\varepsilon}, \mathbf{x}, \mathbf{y}\right) \sim \frac{1}{|\mathbf{x}-\mathbf{y}|^{(d-1) / 2}} e^{i \frac{\omega}{\varepsilon} \frac{|\mathbf{x}-\mathbf{y}|}{c_{0}}}
$$



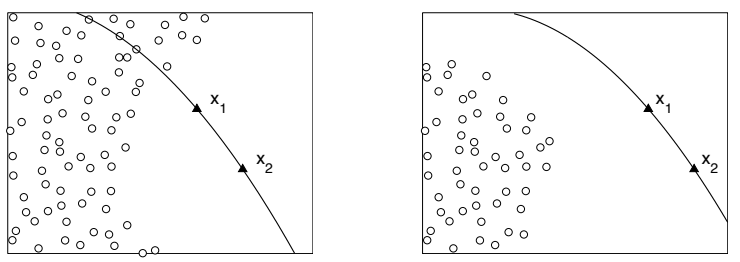

FiguRE 6. If the ray going through $\mathbf{x}_{1}$ and $\mathbf{x}_{2}$ (solid line) enters into the source region (left figure), then the travel time can be estimated from the cross correlation. If this is not the case, then the cross correlation does not have a peak at the travel time (right figure).

For a general smoothly varying background with propagation speed $c_{0}(\mathbf{x})$, the high-frequency behavior of the Green's function is also related to the travel time and it is given by the WKB (Wentzel-Kramers-Brillouin) approximation $[7]$

$$
\hat{G}_{0}\left(\frac{\omega}{\varepsilon}, \mathbf{x}, \mathbf{y}\right) \sim a(\mathbf{x}, \mathbf{y}) e^{i \frac{\omega}{\varepsilon} \tau(\mathbf{x}, \mathbf{y})},
$$

which is valid when $\varepsilon \ll 1$. Here the coefficients $a(\mathbf{x}, \mathbf{y})$ and $\tau(\mathbf{x}, \mathbf{y})$ are smooth except at $\mathbf{x}=\mathbf{y}$. The amplitude $a(\mathbf{x}, \mathbf{y})$ satisfies a transport equation and the travel time $\tau(\mathbf{x}, \mathbf{y})$ satisfies the eikonal equation. It is a symmetric function $\tau(\mathbf{x}, \mathbf{y})=\tau(\mathbf{y}, \mathbf{x})$ and it can be obtained from Fermat's principle

$$
\tau(\mathbf{x}, \mathbf{y})=\inf \left\{T \text { s.t. } \exists\left(\mathbf{X}_{t}\right)_{t \in[0, T]} \in \mathcal{C}^{1}, \mathbf{X}_{0}=\mathbf{x}, \mathbf{X}_{T}=\mathbf{y},\left|\frac{d \mathbf{X}_{t}}{d t}\right|=c_{0}\left(\mathbf{X}_{t}\right)\right\} .
$$

A curve $\left(\mathbf{X}_{t}\right)_{t \in[0, T]}$ that produces the minimum in (23) is a ray and it satisfies Hamilton's equations (29-30). For simplicity we assume that the background speed $c_{0}(\mathbf{x})$ is such that there is a unique ray joining any pair of points $(\mathbf{x}, \mathbf{y})$ in the region of interest.

We can now describe the behavior of the cross correlation function between $\mathbf{x}_{1}$ and $\mathbf{x}_{2}$ when $\varepsilon$ is small, with and without directional energy flux from the sources.

Proposition 6.1. As $\varepsilon$ tends to zero, the cross correlation $C^{(1)}\left(\tau, \mathbf{x}_{1}, \mathbf{x}_{2}\right)$ has singular components if and only if the ray going through $\mathbf{x}_{1}$ and $\mathbf{x}_{2}$ reaches into the source region, that is, into the support of the function $\theta$. In this case there are either one or two singular components at $\tau= \pm \tau\left(\mathbf{x}_{1}, \mathbf{x}_{2}\right)$.

More precisely, any ray going from the source region to $\mathbf{x}_{2}$ and then to $\mathbf{x}_{1}$ gives rise to a singular component at $\tau=-\tau\left(\mathbf{x}_{1}, \mathbf{x}_{2}\right)$. Rays going from the source region to $\mathbf{x}_{1}$ and then to $\mathbf{x}_{2}$ give rise to a singular component at $\tau=\tau\left(\mathbf{x}_{1}, \mathbf{x}_{2}\right)$.

This proposition explains why travel time estimation is bad when the ray joining $\mathbf{x}_{1}$ and $\mathbf{x}_{2}$ is roughly perpendicular to the direction of the energy flux from the noise sources, as in the right of Figure 6 . Its proof is given in $[20]$ and it is based on a stationary phase argument that we sketch out below.

Proof. Using (13) we have

$$
C^{(1)}\left(\tau, \mathbf{x}_{1}, \mathbf{x}_{2}\right)=\frac{1}{2 \pi} \int d \mathbf{y} \int d \omega \hat{G}_{0}\left(\frac{\omega}{\varepsilon}, \mathbf{x}_{1}, \mathbf{y}\right) \hat{G}_{0}\left(\frac{\omega}{\varepsilon}, \mathbf{x}_{2}, \mathbf{y}\right) e^{-i \frac{\omega}{\varepsilon} \tau} \hat{F}(\omega) \theta(\mathbf{y}) .
$$

First we use the WKB approximation (22) of the Green's function and obtain

$$
C^{(1)}\left(\tau, \mathbf{x}_{1}, \mathbf{x}_{2}\right)=\frac{1}{2 \pi} \int d \mathbf{y} \theta(\mathbf{y}) \int d \omega \hat{F}(\omega) \bar{a}\left(\mathbf{x}_{1}, \mathbf{y}\right) a\left(\mathbf{x}_{2}, \mathbf{y}\right) e^{i \frac{\omega}{\varepsilon} \mathcal{T}(\mathbf{y})},
$$


where the rapid phase is

$$
\omega \mathcal{T}(\mathbf{y})=\omega\left[\tau\left(\mathbf{x}_{2}, \mathbf{y}\right)-\tau\left(\mathbf{x}_{1}, \mathbf{y}\right)-\tau\right] .
$$

By the stationary phase method [7], the dominant contribution comes from the stationary points $(\omega, \mathbf{y})$ of the phase which satisfy

This implies that

$$
\partial_{\omega}(\omega \mathcal{T}(\mathbf{y}))=0, \quad \nabla_{\mathbf{y}}(\omega \mathcal{T}(\mathbf{y}))=\mathbf{0}
$$

$$
\tau\left(\mathbf{y}, \mathbf{x}_{2}\right)-\tau\left(\mathbf{y}, \mathbf{x}_{1}\right)=\tau, \quad \nabla_{\mathbf{y}} \tau\left(\mathbf{y}, \mathbf{x}_{2}\right)=\nabla_{\mathbf{y}} \tau\left(\mathbf{y}, \mathbf{x}_{1}\right) .
$$

The second condition requires that $\mathbf{x}_{1}$ and $\mathbf{x}_{2}$ lie on the same side of a ray issuing from a point $\mathbf{y}$. If the points are aligned along the ray as $\mathbf{y} \rightarrow \mathbf{x}_{1} \rightarrow \mathbf{x}_{2}$, then the first condition is equivalent to $\tau=\tau\left(\mathbf{x}_{1}, \mathbf{x}_{2}\right)$. If the points are aligned along the ray as $\mathbf{y} \rightarrow \mathbf{x}_{2} \rightarrow \mathbf{x}_{1}$, then the first condition is equivalent to $\tau=-\tau\left(\mathbf{x}_{1}, \mathbf{x}_{2}\right)$. The stationary points $\mathbf{y}$ contribute to the integral only if they are in the support of $\theta$, which is the source region. This completes the proof of the proposition.

\section{EMERGENCE of THE GREEN'S FUnCTION FOR A LOCALIZED DiSTRIBUtion OF SOURCES IN AN ERGODIC CAVITY}

In the case of a spatially localized distribution of noise sources, directional diversity of the recorded fields can be enhanced if there is sufficient scattering in the medium. An ergodic cavity with a homogeneous or inhomogeneous interior is a good example (Figure 4, left): Even with a source distribution that has very limited spatial support, the reverberations of the waves in the cavity generate interior fields with high directional diversity $[3,14]$. In this section we consider the damped wave equation

$$
\left(\frac{1}{T_{a}}+\frac{\partial}{\partial t}\right)^{2} u-\nabla_{\mathbf{x}} \cdot\left[c^{2}(\mathbf{x}) \nabla_{\mathbf{x}}\right] u=c^{2}(\mathbf{x}) n^{\varepsilon}(t, \mathbf{x})
$$

in a bounded domain $\Omega$ with Dirichlet boundary conditions on $\partial \Omega$. Semiclassical analysis is a very efficient tool to study wave propagation in an ergodic cavity with a smoothly varying background velocity $c(\mathbf{x})$. Note that a wave equation with a self-adjoint operator is considered in (25) in order to simplify the algebra, but the result could be extended to more general wave equations. The use of semi-classical analysis does not allow us to assume that the noise sources are delta-correlated in space. It requires a source distribution with spatial correlation. Therefore, we here assume that the spatial covariance function has the form

$$
\Gamma^{\varepsilon}(\mathbf{x}, \mathbf{y})=\theta\left(\frac{\mathbf{x}+\mathbf{y}}{2}, \frac{\mathbf{x}-\mathbf{y}}{\varepsilon}\right) .
$$

Here the spatial correlation radius of the noise sources is assumed to be of the same order as the decoherence time $(\varepsilon)$, which is the regime in which time and space noise correlations contribute to the Green's function estimation at the same order of magnitude.

The covariance operator $\Theta: L^{2}(\Omega) \rightarrow L^{2}(\Omega)$ defined by

$$
\Theta \psi(\mathbf{x})=\int \Gamma^{\varepsilon}(\mathbf{x}, \mathbf{y}) \psi(\mathbf{y}) d y
$$

is a zero-order pseudodifferential operator with symbol $\hat{\theta}(\mathbf{x}, \boldsymbol{\xi})$

$$
\Theta=\mathrm{Op}^{\varepsilon}[\hat{\theta}(\mathbf{x}, \boldsymbol{\xi})],
$$

where the Fourier transform $\hat{\theta}(\mathbf{x}, \boldsymbol{\xi})$ of the function $\mathbf{z} \mapsto \theta(\mathbf{x}, \mathbf{z})$ is

$$
\hat{\theta}(\mathbf{x}, \boldsymbol{\xi})=\int \theta(\mathbf{x}, \mathbf{z}) e^{-i \boldsymbol{\xi} \cdot \mathbf{z}} d \mathbf{z}
$$


and we have used the Weyl quantization $\mathrm{Op}^{\varepsilon}$ defined by

$$
\mathrm{Op}^{\varepsilon}[\hat{\theta}(\mathbf{x}, \boldsymbol{\xi})] \psi(\mathbf{x})=\frac{1}{(2 \pi)^{d}} \iint \hat{\theta}\left(\frac{\mathbf{x}+\mathbf{y}}{2}, \boldsymbol{\xi}\right) e^{\frac{i}{\varepsilon} \boldsymbol{\xi} \cdot(\mathbf{x}-\mathbf{y})} \psi(\mathbf{y}) d \mathbf{y} d \boldsymbol{\xi}
$$

The main result of the papers $[3,14]$ is that it is possible to reconstruct the singular components of the Green's function in the ergodic case, up to a smoothing operator that depends on $\Gamma^{\varepsilon}$ and $F^{\varepsilon}$. There are two analytical facts that are used in this result:

1) Approximation of full wave propagation by classical ray dynamics (Egorov theorem): the singular (highfrequency) components propagate along the rays $\left(\mathbf{X}_{t}, \boldsymbol{\xi}_{t}\right)$ of geometric optics defined by

$$
\begin{aligned}
\frac{d \mathbf{X}_{t}}{d t} & =c^{2}\left(\mathbf{X}_{t}\right) \boldsymbol{\xi}_{t}, \quad \mathbf{X}_{0}(\mathbf{x}, \boldsymbol{\xi})=\mathbf{x}, \\
\frac{d \boldsymbol{\xi}_{t}}{d t} & =-\frac{1}{2} \nabla\left[c^{2}\right]\left(\mathbf{X}_{t}\right)\left|\boldsymbol{\xi}_{t}\right|^{2}, \quad \boldsymbol{\xi}_{0}(\mathbf{x}, \boldsymbol{\xi})=\boldsymbol{\xi},
\end{aligned}
$$

and with specular reflection at the boundary $\partial \Omega$.

2) Ergodicity of the ray dynamics in the cavity $\Omega$ : This means that, starting from almost any point $\mathbf{x}$ and almost any direction $\boldsymbol{\xi}$, the ray $\left(\mathbf{X}_{t}, \boldsymbol{\xi}_{t}\right)$ visits all of phase space.

Proposition 7.1. If $c \in W^{4, \infty}(\Omega), \hat{\theta}$ is smooth, bounded, and integrable, then $\partial_{\tau} C^{(1)}(\tau, \mathbf{x}, \mathbf{y})$ is the kernel of the operator

$$
e^{-\frac{|\tau|}{T_{a}}} K_{\theta}^{\varepsilon}\left[F^{\varepsilon} * G(\tau)-F^{\varepsilon} * G(-\tau)\right]+R^{\varepsilon}(\tau)+R_{T_{a}}(\tau),
$$

for any $\tau>0$, where $G(\tau)$ is the Green's function operator with kernel $G(\tau, \mathbf{x}, \mathbf{y})$ and $K_{\theta}^{\varepsilon}$ is the smoothing operator

$$
K_{\theta}^{\varepsilon}=\mathrm{Op}^{\varepsilon}\left[\hat{k}_{\theta}(c(\mathbf{x}) \boldsymbol{\xi})\right], \quad \hat{k}_{\theta}(\tilde{\boldsymbol{\xi}})=\frac{\int_{\Omega} d \mathbf{z} c(\mathbf{z})^{-d} \int_{\partial B(\mathbf{0}, 1)} d S(\boldsymbol{\eta}) \hat{\theta}\left(\mathbf{z},|\tilde{\boldsymbol{\xi}}| \frac{\boldsymbol{\eta}}{c(\mathbf{z})}\right)}{\int_{\Omega} c(\mathbf{z})^{-d} d \mathbf{z} \int_{\partial B(\mathbf{0}, 1)} d S(\boldsymbol{\eta})} .
$$

The remainder $R^{\varepsilon}(\tau)$ is determined by the error in the semiclassical approximation and it is small if $\varepsilon$ is small. The remainder $R_{T_{a}}(\tau)$ is determined by the rate of convergence of the ergodic theorem for the function $\hat{\theta}$ of the classical Hamiltonian flow. If denote by $T_{\mathrm{erg}}$ the characteristic convergence time of $\frac{1}{t} \int_{0}^{t} \hat{\theta}\left(\mathbf{X}_{s}, \boldsymbol{\xi}_{s}\right) d s$ to its ergodic limit, then $R_{T_{a}}(\tau)$ is small if $T_{a} \gg T_{\mathrm{erg}}$.

The symbol of the smoothing operator $K_{\theta}^{\varepsilon}$ is $\hat{k}_{\theta}(c(\mathbf{x}) \boldsymbol{\xi})$. The form of the symbol of $K_{\theta}^{\varepsilon}$ is obtained by averaging the symbol $\hat{\theta}(\mathbf{x}, \boldsymbol{\xi})$ of the covariance operator $\Theta$ over the Liouville measure on surfaces of constant energy. This makes sense intuitively since, in the semiclassical limit, we can expect the symbol of $K_{\theta}^{\varepsilon}$ to be close to the one of $\Theta$ transported by the classical Hamiltonian flow, and this converges to (32) by the ergodic theorem. This shows that the support of the smoothing operator $K_{\theta}^{\varepsilon}$ has an effective radius that is of the order of the correlation radius of the sources. To summarize, if $T_{\operatorname{erg}} \ll T_{a}$ and $\varepsilon \ll 1$, then detecting the first peak of $\tau \mapsto C^{(1)}\left(\tau, \mathbf{x}_{1}, \mathbf{x}_{2}\right)$ gives an estimate of the travel time from $\mathbf{x}_{1}$ to $\mathbf{x}_{2}$. The accuracy of this estimate depends on the correlation radius and the decoherence time of the noise sources.

\section{ITERATED CROSS CORRELATIONS FOR TRAVEL TIME ESTIMATION IN A WEAKLY SCATTERING MEDIUM}

For travel time tomography to be successful it is necessary to have good estimates of the travel times between pairs of sensors that cover well the region of interest. When the noise sources are spatially localized and there is a strong directional energy flux at the sensors, then travel time estimates will be poor for sensor pairs with axis in directions perpendicular to this flux. In this section we show that it is possible to exploit scattering from random inhomogeneities so as to enhance travel time estimation. 
We consider distributions of noise sources that are spatially localized and media with scattering that is not strong enough for equipartition of the fields at the sensors [36]. Therefore, even with scattering, the signals depend strongly on the spatial localization of the noise sources, which affects the quality of travel time estimation. However, the coda (i.e. the tails) of the cross correlations are generated by scattered waves, which have more directional diversity than the direct waves from the noise sources. By cross correlating the coda of the cross correlations, which produces fourth-order cross correlations, it is possible to exploit scattered waves and their enhanced directional diversity. Campillo and Stehly [12] suggest a way to estimate the Green's function between $\mathbf{x}_{1}$ and $\mathbf{x}_{2}$ as follows.

1) Calculate the cross correlations between $\mathbf{x}_{1}$ and $\mathbf{x}_{\mathrm{a}, j}$ and between $\mathbf{x}_{2}$ and $\mathbf{x}_{\mathrm{a}, j}$ for a large number $N$ of auxiliary sensors $\left(\mathbf{x}_{\mathrm{a}, j}\right)_{j=1, \ldots, N}$ that are distributed over the medium

$$
C_{T}\left(\tau, \mathbf{x}_{\mathrm{a}, j}, \mathbf{x}_{l}\right)=\frac{1}{T} \int_{0}^{T} u\left(t, \mathbf{x}_{\mathrm{a}, j}\right) u\left(t+\tau, \mathbf{x}_{l}\right) d t, \quad l=1,2 .
$$

2) Cross correlate the coda of these cross correlations and sum them over all auxiliary sensors to form the coda cross correlation between $\mathbf{x}_{1}$ and $\mathbf{x}_{2}$ :

$$
C_{T^{\prime}, T}\left(\tau, \mathbf{x}_{1}, \mathbf{x}_{2}\right)=\sum_{j=1}^{N} \int_{\left[-T^{\prime},-T_{\text {coda }}\right] \cup\left[T_{\text {coda }}, T^{\prime}\right]} C_{T}\left(\tau^{\prime}, \mathbf{x}_{\mathrm{a}, j}, \mathbf{x}_{1}\right) C_{T}\left(\tau^{\prime}+\tau, \mathbf{x}_{\mathrm{a}, j}, \mathbf{x}_{2}\right) d \tau^{\prime}
$$

Here the time $T_{\text {coda }}$ is chosen so as to eliminate the coherent part of $C_{T}$. In general, $T_{\text {coda }}$ depends on $j$ and should be chosen larger than $\max \left(\tau\left(\mathbf{x}_{\mathrm{a}, j}, \mathbf{x}_{1}\right), \tau\left(\mathbf{x}_{\mathrm{a}, j}, \mathbf{x}_{2}\right)\right)$.

Using the stationary phase method we have shown in [20] that the algorithm proposed by Campillo and Stehly succeeds in exploiting the enhanced directivity of scattered waves. We have shown in particular that the coda cross correlation $C_{T^{\prime}, T}$, defined by (33), has singular components at the travel time between the sensors even in the unfavorable case in which the ray joining $\mathbf{x}_{1}$ and $\mathbf{x}_{2}$ does not reach into the source region, as discussed in Section 6. This result is presented in Proposition 8.2 below. Its proof requires to specify a model for the inhomogeneous medium. A simple, single-scattering model is sufficient for this purpose. We assume that the medium has a smoothly varying background speed $c_{0}(\mathbf{x})$, which we want to image, and a large collection of point scatterers at $\left(\mathbf{z}_{\mathrm{s}, j}\right)_{j \geq 1}$. Their reflectivities $\sigma_{j}$ are assumed to be independent random variables with zero-mean and with small variance $\sigma^{2}$. In the Born (single-scattering) approximation the full Green's function at scaled high frequencies is given by

$$
\hat{G}\left(\frac{\omega}{\varepsilon}, \mathbf{x}, \mathbf{y}\right)=\hat{G}_{0}\left(\frac{\omega}{\varepsilon}, \mathbf{x}, \mathbf{y}\right)+\hat{G}_{1}\left(\frac{\omega}{\varepsilon}, \mathbf{x}, \mathbf{y}\right)
$$

where $\hat{G}_{0}$ is the Green's function (19) of the background medium and $\hat{G}_{1}$ is given by

$$
\hat{G}_{1}\left(\frac{\omega}{\varepsilon}, \mathbf{x}, \mathbf{y}\right)=\omega^{2} \sum_{j} \sigma_{j} \hat{G}_{0}\left(\frac{\omega}{\varepsilon}, \mathbf{x}, \mathbf{z}_{\mathrm{s}, j}\right) \hat{G}_{0}\left(\frac{\omega}{\varepsilon}, \mathbf{z}_{\mathrm{s}, j}, \mathbf{y}\right) .
$$

We also assume that:

1) The auxiliary sensors at $\left(\mathbf{x}_{\mathrm{a}, j}\right)_{j=1, \ldots, N}$ are distributed with a continuum density $\chi_{\mathrm{a}}\left(\mathbf{x}_{\mathrm{a}}\right)$. This means that we can approximate sums over the auxiliary sensors by integrals with density $\chi_{\mathrm{a}}$ :

$$
\sum_{j} \psi\left(\mathbf{x}_{\mathrm{a}, j}\right) \simeq \int d \mathbf{x}_{\mathrm{a}} \chi_{\mathrm{a}}\left(\mathbf{x}_{\mathrm{a}}\right) \psi\left(\mathbf{x}_{\mathrm{a}}\right)
$$

for any real-valued test function $\psi$. The support of the function $\chi_{\text {a }}$ defines the support of the region of the auxiliary sensors. 


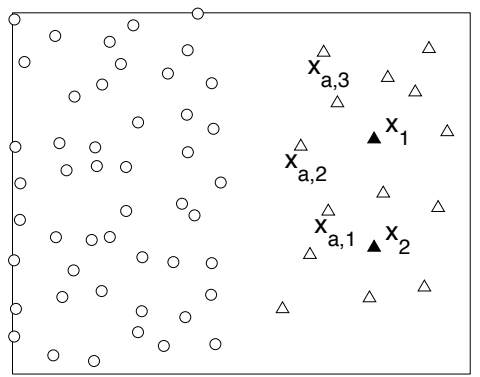

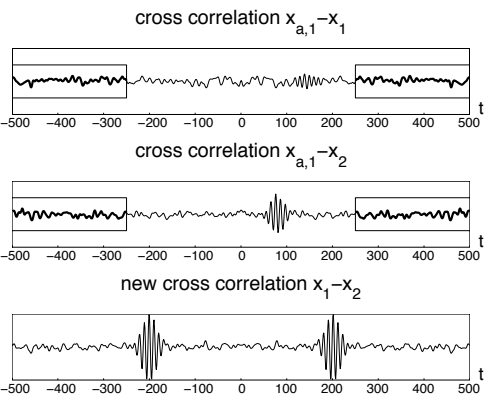

FiguRE 7. When noise sources (circles in the left figure) are spatially localized, we form cross correlations between the recorded signals at $\mathbf{x}_{1}$ and $\mathbf{x}_{2}$ (solid triangles) and those at auxiliary sensors at $\mathbf{x}_{\mathrm{a}, j}$ (empty triangles). We then compute cross correlations of the tails of these correlations (here, $T_{\text {coda }}=250$ ) and sum over the auxiliary sensors. This gives the coda cross correlation (33) from which the travel time between $\mathbf{x}_{1}$ and $\mathbf{x}_{2}$ can be better estimated, as indicated in the schematic figure on the right.

2) The scatterers at $\left(\mathbf{z}_{\mathrm{s}, j}\right)_{j \geq 1}$ are distributed with a continuum density $\chi_{\mathrm{s}}\left(\mathbf{z}_{\mathrm{s}}\right)$. The support of the function $\chi_{\mathrm{s}}$ defines the support of the scattering region.

We distinguish two components in the cross correlation function $C^{(1)}$ defined by (11):

1) The coherent component $C_{\text {coh }}^{(1)}$ is associated with the direct waves that have not been scattered. From the representation (34) of the Green's function, the coherent component $C_{\mathrm{coh}}^{(1)}$ of the cross correlation function is given by

$$
C_{\mathrm{coh}}^{(1)}\left(\tau, \mathbf{x}_{1}, \mathbf{x}_{2}\right)=\int d \mathbf{y} \theta(\mathbf{y}) \int d s d s^{\prime} F^{\varepsilon}\left(s^{\prime}\right) G_{0}\left(s, \mathbf{x}_{1}, \mathbf{y}\right) G_{0}\left(\tau+s+s^{\prime}, \mathbf{x}_{2}, \mathbf{y}\right),
$$

where $G_{0}$ is the Green's function of the smoothly varying background.

2) The remaining coda component $C_{\mathrm{coda}}^{(1)}$, which is the contribution of the waves scattered by the inhomogeneities:

$$
C_{\text {coda }}^{(1)}\left(\tau, \mathbf{x}_{1}, \mathbf{x}_{2}\right)=C^{(1)}\left(\tau, \mathbf{x}_{1}, \mathbf{x}_{2}\right)-C_{\mathrm{coh}}^{(1)}\left(\tau, \mathbf{x}_{1}, \mathbf{x}_{2}\right) .
$$

We consider first the coherent fourth-order cross correlation function

$$
C^{(2)}\left(\tau, \mathbf{x}_{1}, \mathbf{x}_{2}\right)=\lim _{T^{\prime} \rightarrow \infty} \int_{-T^{\prime}}^{T^{\prime}} d \tau^{\prime} \sum_{j} C_{\mathrm{coh}}^{(1)}\left(\tau^{\prime}, \mathbf{x}_{\mathrm{a}, j}, \mathbf{x}_{1}\right) C_{\mathrm{coh}}^{(1)}\left(\tau^{\prime}+\tau, \mathbf{x}_{\mathrm{a}, j}, \mathbf{x}_{2}\right) .
$$

The following proposition is analogous to Proposition 6.1.

Proposition 8.1. The cross correlation $C^{(2)}\left(\tau, \mathbf{x}_{1}, \mathbf{x}_{2}\right)$ has singular components if and only if the ray going through $\mathbf{x}_{1}$ and $\mathbf{x}_{2}$ extends into the source region. If this is the case, then there are one or two singular components at $\tau= \pm \tau\left(\mathbf{x}_{1}, \mathbf{x}_{2}\right)$.

We see, therefore, that there is no gain in using the function $C^{(2)}$ in place of $C^{(1)}$ for travel time estimation, the singular component of the coherent fourth-order cross correlation $C^{(2)}$ has the same properties as the one of the standard cross correlation $C^{(1)}$. We now consider the coda cross correlation given by

$$
C^{(3)}\left(\tau, \mathbf{x}_{1}, \mathbf{x}_{2}\right)=\lim _{T^{\prime} \rightarrow \infty} \int_{-T^{\prime}}^{T^{\prime}} d \tau^{\prime} \sum_{j} C_{\mathrm{coda}}^{(1)}\left(\tau^{\prime}, \mathbf{x}_{\mathrm{a}, j}, \mathbf{x}_{1}\right) C_{\mathrm{coda}}^{(1)}\left(\tau^{\prime}+\tau, \mathbf{x}_{\mathrm{a}, j}, \mathbf{x}_{2}\right) .
$$




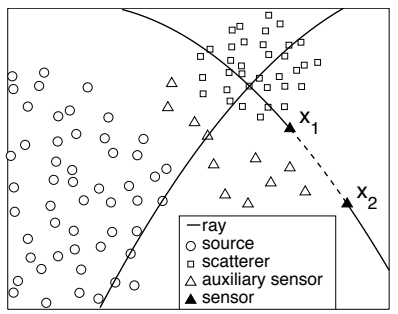

FIgURE 8. Schematic of a configuration of noise sources (circles), scatterers (squares), and auxiliary sensors (empty triangles) that give enhanced travel time estimation between $\mathbf{x}_{1}$ and $\mathbf{x}_{2}$ (solid triangles). First, there are scatterers along the ray going through $\mathbf{x}_{1}$ and $\mathbf{x}_{2}$ (but the scatterers along the segment between $\mathbf{x}_{1}$ and $\mathbf{x}_{2}$ play no role). Second, there are auxiliary sensors along rays going from the source region into the scatterer region.

Proposition 8.2. There are two (and only two) singular components in $C^{(3)}$, at times $\tau= \pm \tau\left(\mathbf{x}_{1}, \mathbf{x}_{2}\right)$, if the two following conditions hold (see Figure 8):

1) The ray going through $\mathbf{x}_{1}$ and $\mathbf{x}_{2}$ (excluding the segment between $\mathbf{x}_{1}$ and $\mathbf{x}_{2}$ ) reaches into the scattering region (the support of $\chi_{\mathrm{s}}$ ). The scatterers along this ray are the basic ones for enhanced travel time estimation.

2) Rays going from the source region (the support of the function $\theta$ ) to the basic scatterers reach into the observation region (the support of the function $\chi_{\mathrm{a}}$ ).

The important point here is that it is not required that the ray joining the sensors $\mathbf{x}_{1}$ and $\mathbf{x}_{2}$ should extend into the source region as in Propositions 6.1 and 8.1. Therefore the configurations for which the coda cross correlation $C^{(3)}$ has a singular component at the travel time between $\mathbf{x}_{1}$ and $\mathbf{x}_{2}$ are much more general than the ones for which the standard cross correlation has a singular component. Propositions 8.1 and 8.2 show that there is no additional information about the travel time in the coherent fourth-order cross correlation, but that there is such information in the coda (fourth-order) cross correlation. Therefore, the coda cross correlation is the one that should be used. The full fourth-order cross correlation function, obtained by cross-correlating the full cross correlation functions $C^{(1)}=C_{\text {coh }}^{(1)}+C_{\text {coda }}^{(1)}$, is the sum of the coherent fourth-order cross correlation $C^{(2)}$, the coda fourth-order cross correlation $C^{(3)}$, and cross correlations between the coherent $C_{\text {coh }}^{(1)}$ and the coda $C_{\text {coda }}^{(1)}$. Using again the stationary phase method, we can see that these cross correlations have singular components only at $\tau= \pm \tau\left(\mathbf{x}_{1}, \mathbf{x}_{2}\right)$, and only if the ray joining $\mathbf{x}_{1}$ and $\mathbf{x}_{2}$ extends into the source region. Therefore, while the interesting singular component of the coda fourth-order cross correlation is present in the full fourth-order cross correlation, it is buried in many other uninteresting terms, especially when the scattered waves are much weaker than the direct waves. That is why it is much better, in terms of the signal-to-noise ratio, to use only the coda fourth-order cross correlations.

\section{REFERENCES}

[1] Abramowitz M and Stegun I 1965 Handbook of mathematical functions (New York: Dover Publications)

[2] Aki K and Chouet B 1975 Origin of coda waves: Source, attenuation and scattering effects J. Geophys. Res. 80 3322-3342

[3] Bardos C, Garnier J, and Papanicolaou G 2008 Identification of Green's functions singularities by cross correlation of noisy signals Inverse Problems 24015011

[4] Berryman J 1990 Stable iterative reconstruction algorithm for nonlinear travel time tomography Inverse Problems 6 21-42

[5] Biondi B L 2006 3D Seismic imaging no. 14 in Investigations in Geophysics (Tulsa: Society of Exploration Geophysics)

[6] Blackstock D T 2000 Fundamentals of physical acoustics (New York: Wiley)

[7] Bleistein N, Cohen J K and Stockwell J W Jr 2001 Mathematics of multidimensional seismic imaging, migration, and inversion (New York: Springer Verlag)

[8] Born M and Wolf E 1999 Principles of optics (Cambridge: Cambridge University Press) 
[9] Brenguier F, Shapiro N M, Campillo M, Ferrazzini V, Duputel Z, Coutant O, and Nercessian A 2008 Towards forecasting volcanic eruptions using seismic noise Nature Geoscience 1 126-130

[10] Brenguier F, Shapiro N M, Campillo M, Nercessian A, and Ferrazzini V 2007 3-D surface wave tomography of the Piton de la Fournaise volcano using seismic noise correlations Geophys. Res. Lett. 34 L02305

[11] Campillo M and Paul A 2003 Long range correlations in the seismic coda Science 29 547-549

[12] Campillo M and Stehly L 2007 Using coda waves extracted from microseisms to construct direct arrivals Eos Trans. AGU 88(52), Fall Meet. Suppl., Abstract S51D-07

[13] Claerbout J F 1985 Imaging the Earth's interior (Palo Alto: Blackwell Scientific Publications)

[14] Colin de Verdière Y 2006 Mathematical models for passive imaging. I: general background arXiv:math-ph/0610043v1

[15] Colin de Verdière Y 2006 Mathematical models for passive imaging. II: Effective Hamiltonians associated to surface waves arXiv:math-ph/0610044v1

[16] Courant R and Hilbert D 1991 Methods of mathematical physics Vol. I and II (New York: Wiley)

[17] Curtis A, Gerstoft P, Sato H, Snieder R, and Wapenaar K 2006 Seismic interferometry - turning noise into signal The Leading Edge 25 1082-1092

[18] Duvall Jr T L, Jefferies S M, Harvey J W, and Pomerantz M A 1993 Time-distance helioseismology Nature 362 $430-432$

[19] Fouque J P, Garnier J, Papanicolaou G, and Sølna K 2007 Wave propagation and time reversal in randomly layered media (New York: Springer)

[20] Garnier J and Papanicolaou G 2008 Passive sensor imaging using cross correlations of noisy signals in a scattering medium submitted available at http://www.proba.jussieu.fr/ garnier/

[21] Gouédard P, Stehly L, Brenguier F, Campillo M, Colin de Verdière Y, Larose E, Margerin L, Roux P, Sanchez-Sesma F J, Shapiro N M, and Weaver R L 2008 Cross-correlation of random fields: mathematical approach and applications Geophysical Prospecting 56 375-393

[22] Larose E, Margerin L, Derode A, Van Tiggelen B, Campillo M, Shapiro N, Paul A, Stehly L, and Tanter M 2006 Correlation of random wave fields: an interdisciplinary review Geophysics $\mathbf{7 1}$ SI11-SI21

[23] Lobkis O I and Weaver R L 2001 On the emergence of the Green's function in the correlations of a diffuse field J. Acoustic. Soc. Am. 110 3011-3017

[24] Malcolm A E, Scales J, and Van Tiggelen B A 2004 Extracting the Green function from diffuse, equipartitioned waves Phys. Rev. E $\mathbf{7 0} 015601$

[25] Rickett J and Claerbout J 1999 Acoustic daylight imaging via spectral factorization: Helioseismology and reservoir monitoring The Leading Edge 18 957-960

[26] Roux P and Fink M 2003 Green's function estimation using secondary sources in a shallow water environment J. Acoust. Soc. Am. 113 1406-1416

[27] Roux P, Sabra K G, Kuperman W A, and Roux A 2005 Ambient noise cross correlation in free space: Theoretical approach J. Acoust. Soc. Am. 117 79-84

[28] Ryzhik L V, Papanicolaou G C, and Keller J B 1996 Transport equations for elastic and other waves in random media Wave Motion 24 327-370

[29] Sabra K G, Gerstoft P, Roux P, and Kuperman W 2005 Surface wave tomography from microseisms in Southern California Geophys. Res. Lett. 32 L14311.

[30] Sabra K G, Roux P, Gerstoft P, Kuperman W A, and Fehler M C 2006 Extracting coherent coda arrivals from cross correlations of long period seismic waves during the Mount St Helen's 2004 eruption Geophys. Res. Lett. 33 L06313

[31] Sato H and Fehler M 1998 Wave propagation and scattering in the heterogeneous Earth (New York: Springer-Verlag)

[32] Schuster G T, Yu J, Sheng J, and Rickett J 2004 Interferometric daylight seismic imaging Geophysical Journal International $157832-852$

[33] Shapiro N and Campillo M 2004 Emergence of broadband rayleigh waves from correlations of the ambient seismic noise Geophys. Res. Lett. 31 L07614

[34] Shapiro N M, Campillo M, Stehly L, and Ritzwoller M H 2005 High-resolution surface wave tomography from ambient noise Science 307 1615-1618

[35] Snieder R 2004 Extracting the Green's function from the correlation of coda waves: A derivation based on stationary phase Phys. Rev. E 69046610

[36] Stehly L, Campillo M, and Shapiro N M 2006 A study of the seismic noise from its long-range correlation properties Geophys. Res. Lett. 111 B10306

[37] Wapenaar K and Fokkema J 2006 Green's function representations for seismic interferometry Geophysics 71 SI33-SI46.

[38] Weaver R and Lobkis O I 2001 Ultrasonics without a source: Thermal fluctuation correlations at MHz frequencies Phys. Rev. Lett. 87134301

[39] Yao H, van der Hilst R D, and de Hoop M V 2006 Surface-wave array tomography in SE Tibet from ambient seismic noise and two-station analysis I. Phase velocity maps Geophysical Journal International 166 732-744 